\title{
Multi-parameter Extraction from SOI Photonic Integrated Circuits using Circuit Simulation and Evolutionary Algorithms
}

\author{
Ruocco A. ${ }^{a}$, Fiers M. ${ }^{b}$,Vanslembrouck M. ${ }^{a}$,Van Vaerenbergh T. ${ }^{a}$, Bogaerts W. ${ }^{a, b}$ \\ ${ }^{a}$ Photonics Research Group, Department of Information Technology, Center of Nano-and \\ Biophotonics, Ghent University - IMEC, Sint-Pietersnieuwstraat 41, 9000 Gent, Belgium \\ ${ }^{b}$ Luceda Photonics, Noordlaan 21, 9200 Dendermonde, Belgium
}

\begin{abstract}
We propose a procedure to extract multiple parameters from the spectral characteristic of a single photonic integrated circuit. We applied the method on high order silicon Mach-Zehnder lattice filters: ${ }^{1}$ these filters are realized by cascading delay stages and directional couplers of different length. Because of their cascaded nature and steep roll-off properties, these devices can be used to accurately extract properties of the waveguides and the directional couplers. The spectral transmission is measured between the inputs and the outputs. This result is compared to a full CAPHE optical circuit simulation with parametric behavioral models for the waveguide and the directional couplers. An evolutionary fitting algorithm based on the covariance matrix adaptation method is used to match the circuit simulation with the measurement. This black box approach gives us fast and accurate parameter extraction with a reduced number of iteration steps. The quadratic error between measurement and simulation of each iteration is used as feedback for the evolutionary algorithm that adapts the test values for the following step. The objective of our analysis is an accurate, wavelength-dependent model for the waveguide group index and the directional couplers. The proposed method has been used for wafer scale parameter extraction. Our fast method makes it possible to extract the parameters in real time, and correlate the functional parameters of the waveguides with process statistics collected during fabrication. The obtained parameters are in substantial agreement with the results of the simulations used in the design, and can be used to further improve behavioral models that correlate the manufacturing process data with the optical performance.
\end{abstract}

Keywords: Simulation of photonic integrated circuits, Characterization of photonic integrated circuits, Optical parameter extraction,Processing flow optimization, Numerical techniques for photonic integrated circuits

\section{INTRODUCTION}

Silicon photonics has the potential to be a key enabler for future network applications. Wafer scale fabrication in CMOS lines allow mass production and reduction of the costs. ${ }^{2}$ A wide variety of software tools are available for the simulation of photonic device, amongst them BPM (Beam Propagation Method), FDTD (Finite-Difference Time Domain) method and FEM (Finite Element Method). ${ }^{3}$ These tools are computation power hungry and yet they cannot predict properly the behavior of many processed devices. The bottleneck does not reside with the simulation accuracy or in design procedure, but rather in sensitivity and variability of silicon photonics. ${ }^{4}$ The high index contrast of silicon allows miniaturization of photonic integrated circuits, but at the same time the sub-micron dimensions of the guiding structures make them very sensitive to fabrication variations. ${ }^{5}$ The direct consequence of such sensitivity is that whenever a wavelength selective device has to be designed, the mismatch between the simulation and the real device is unpredictable and unavoidable. Variations of the optical properties across the wafer give rise to gradient-like performance distributions of the resulting devices. An example of devices that suffer form this effect are FIR (Finite Impulse Response) ${ }^{6}$ and IIR (Infinite Impulse Response) filters: ${ }^{7}$ made up of MZIs (Mach-Zehnder interferometers) and MRR (Micro Ring Resonator) networks,

Further author information: (Send correspondence to A.R.)

A.R.: E-mail: alfonso.ruocco@intec.ugent.be, Telephone: +32-9-264-36-31

W.B.: E-mail: wim.bogaerts@intec.ugent.be 


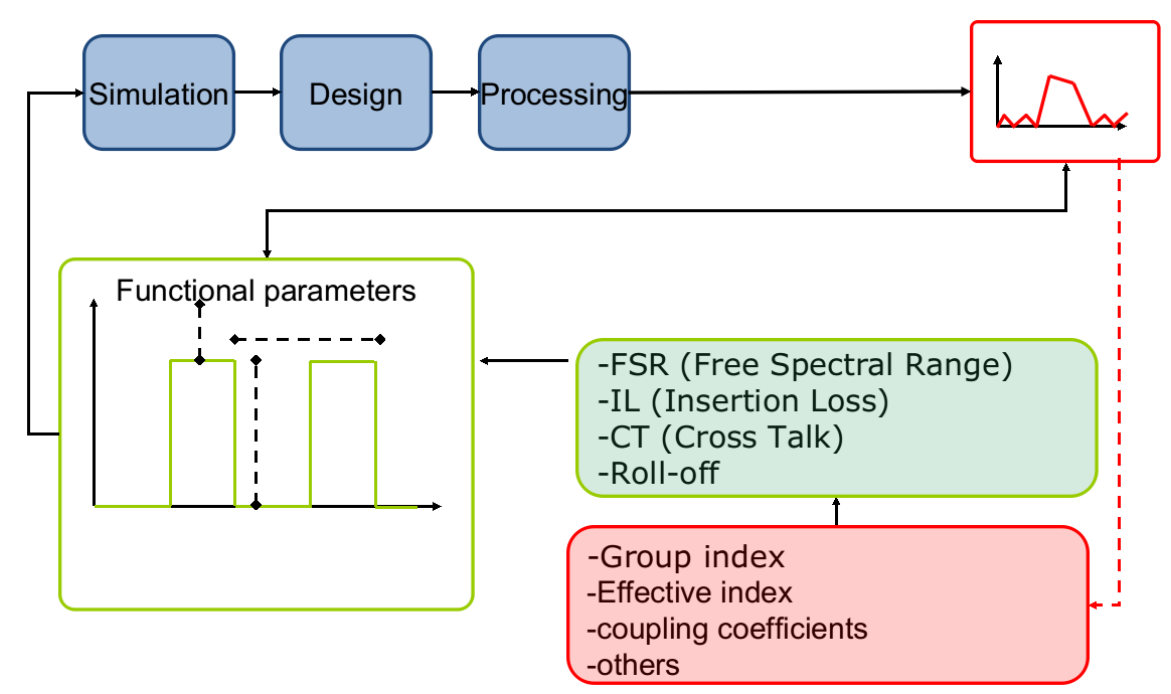

Figure 1: Silicon On Insulator Design Flow

respectively. Fabrication tolerances in thickness and width of photonic waveguides lead to a variation of the FSR (Free Spectral Range), center wavelength position and overall shape of the filter response. Fig.1 describes the commonly used SOI R\&D flow ${ }^{8}$, starting from the required functional parameters and proceeding with design and simulation. The next step is the fabrication carried out in CMOS production lines, and it is followed by the measurement of the device. What is required is a closing loop element to reliably use measurement feedback to calibrate the second generation device and collect variability information to make the device performance more predictable. Instead of compensating for unpredictable variability, an approach is to take it into account in phase of designing. For this, we need to obtain a correlation between the fabrication parameters (and their associated variability) and the device's behavioral parameters. Representative device models and efficient extraction of the model parameters are quintessential to close the design flow reliably. We propose a characterization technique to extract and determine multiple model parameters from a single measurement of a compound photonic integrated circuit. After the design and fabrication, the device is measured and the wavelength response is used as the basis for our parameter extraction technique. The circuit can have multiple inputs and outputs and a combination of responses can be used. The procedure is implemented as a multi-dimensional fitting algorithm where the designed circuit model (with unknown parameters for all building blocks) is fit to the collected transmission measurements. With brute force simulations, the number of iterations in the fit would be too large, while analytical fitting methods would not be sufficiently accurate or cannot capture the correlations between the mathematical coefficients and physical quantities. To satisfy the trade-off condition between these requirements, we adopt an evolutionary strategy combined with an accurate physical modeling of every sub-element in the PIC. We applied this procedure to fourth order silicon lattice filters and correlate the measurement data with the collected process data. ${ }^{3,9}$

\section{LATTICE FILTERS ON SOI PLATFORM}

Lattice filter circuits are the combination of two basic building optical blocks: directional couplers and differential delay sections. A single stage lattice filter with $3 d B$ power splitting of the directional coupler (i.e. a simple Mach-Zehnder interferometer) exhibits a sinusoidal transmission as function of wavelength. As a wavelength filter, this deviceis to limited for most communications applications. One of the possible approach to improve the performance of such filter is to increase the number of cascaded stages. By properly selecting the power splitting ratios of the directional couplers, it is possible to obtain a sum of sinusoidal responses instead of a single sine. Because different coupling ratios give different weight coefficient to the sinusoids, a multi-stage MZI filter acts as a $Z$ transform device. Ideally we can obtain any periodical response that can be described by a $Z$ transform. A rectangular shape filter for example would allow full band use of the filter, but such a response 


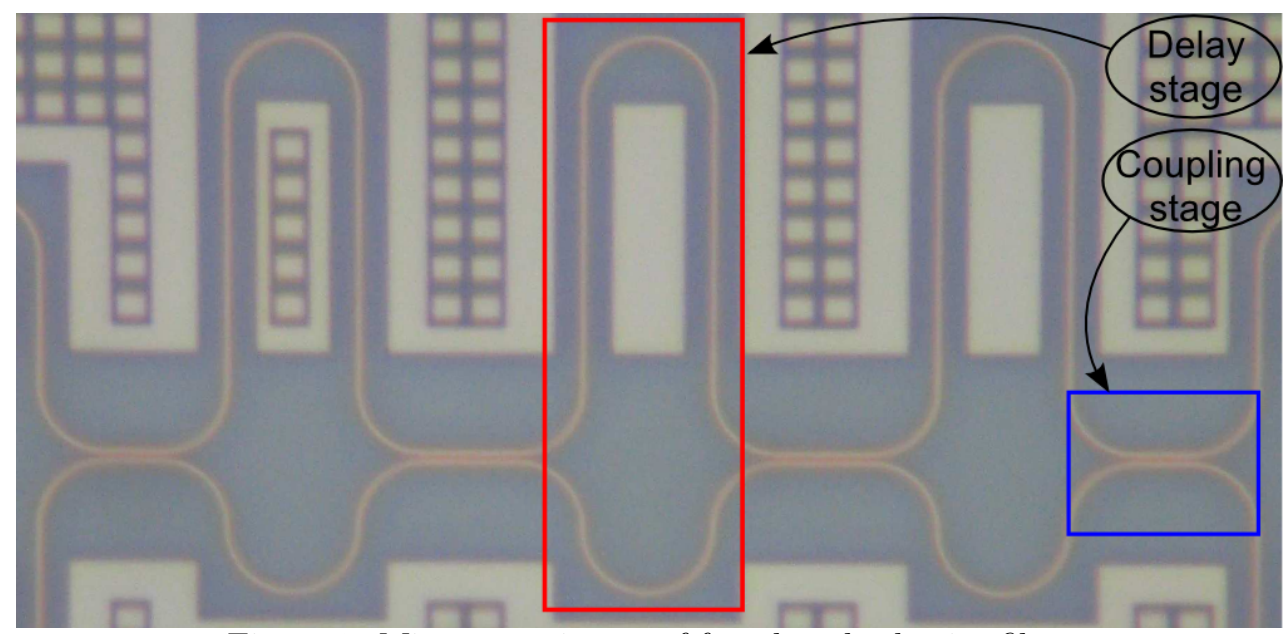

Figure 2: Microscope image of fourth order lattice filter

requires infinite $Z$ transform contributions and therefore infinite stages. With a finite number of cascaded stages, flattened responses are possible, which are needed for many WDM channel filters. Fig.2 shows a microscope image of a four stage lattice filter composed of four directional couplers and three delay stages. In the aforementioned image an example of the delay stage and directional coupler are respectively underlined in the red and blue box.

\subsection{Design Procedure}

The design flow of our MZI lattice starts from the requirements needed for the particular application of the filter: the wavelength response and FSR (Free Spectra Range). The wavelength domain response is determined by the coupling ratios of the directional couplers. We used techniques from digital filter synthesis to calculate the required coupling coefficients. ${ }^{1}$ Different filters shapes are available. Depending on the required bandwidth, roll-off, pass/stop band relations the proper design procedure is chosen, in our case for a Chebyshev filter. This particular filtering window has the property of minimizing the error between ideal and actual filter response over its spectrum. Filter design techniques are generalized, which means that when the coupling ratios are calculated, is not strictly required to know if the field of application is electronics, digital data analysis or photonics. Also, the simulation of the resulting filter can be generalized and represented in normalized frequency from 0 to 1 . Once the spectral response is defined, these have to be translated in actual design parameters specific to the technology. For our silicon photonic filters we choose the wavelength domain and the sub-blocks to be used. The FSR is determined by the delay stage: the optical path length difference between one waveguide and the other is inversely proportional to the FSR of the filter. The optical path length itself is the product of group index and physical length of the guiding structure. The specification for the Chebyshev windowing are 0.25 in normalized frequency, a $20 d B$ stop-band, and $12 \mathrm{~nm}$ free spectral range.

\subsection{SOI Implementation}

The next step in the implementation of our filter in a silicon photonics platform is the selection of the waveguide. Thin silicon waveguides of $220 \mathrm{~nm}$ hight and $450 \mathrm{~nm}$ width are used. The delay section is obtained using two waveguide with matched bend sections and differential straight sections, with a well-controlled difference in physical path length. The bend radius is $5 \mu \mathrm{m}$, which yields acceptably low reflections and losses while keeping the footprint of the whole device small. The coupling sections are simple directional couplers with a pair of parallel identical waveguides. The coupling coefficients of the individual coupling sections is adjusted by changing the length of the directional couplers. The gap between the waveguides is fixed to $180 \mathrm{~nm}$.

\subsection{Simulation of the Filter}

The abstract, generalized filter is simulated using $Z$ transform algorithm. But this method cannot capture the photonic-specific non-idealities. Therefore, we feed the filter specs into the CAPHE circuit simulator. ${ }^{10}$ Each of the sub-blocks (waveguides and directional couplers) is described by its characteristic scatter matrix, and the 
elements of the matrix are calculated using a behavioral model which we detail in section 3 . Waveguides have a $2 \times 2$ scatter matrix, and directional couplers a $4 \times 4$ matrix. However, we ignore reflections in this process, which simplifies the matrices somewhat. However, this simplification has no effect on our method, which would work just as well with reflections. The waveguide and directional coupler blocks are connected together into a network. A wavelength sweep is performed for the simulation of the full spectrum response around the central working wavelength. The scattering matrix elements are extremely flexible, indeed it is possible to have $n$-dimensional $S$ elements. Due to this flexibility any kind of dependency can be added and simulated.

\section{BASIC SUB-BLOCK MODELING}

The sub-blocks used for the implementation of the lattice filter are the delay stages and the directional couplers. These elements can be considered themselves as combinations of more basic devices. The delay stage realized with a pair of optically uncoupled waveguides, while the directional coupler is realized with a pair of waveguides optically coupled and able to split the power in a controllable way. In this section we give a description of the models we use to describe the waveguides and the directional couplers sufficiently accurate in the CAPHE circuit simulator.

\subsection{Delay Stage}

The photonic wire waveguides used for this design support a single TE-like mode, and a single TM-like mode. In this paper, we operate the circuit in the TE polarization and we ignore coupling between polarizations. The waveguide mode is characterized by its effective index $n_{\text {eff }}$. Since the high contrast makes silicon wires a quite dispersive medium, the effective index describes the optical behavior of the waveguide only at a fixed wavelength. To define reliable optical properties of the wire, we require the group index $n_{g}$ and its derivative with wavelength (second order dispersion, or group velocity dispersion) as well.

Equation (1) express the relation used for the modeling of the waveguide optical properties. The effective index is in this case $n_{e f f}(\lambda)$. The $n_{\text {eff }}$ and the $n_{g}$ at lambda zero are simulated with mode solver software. During the initial filter design phase, the derivative of $n_{g}$ is assumed to be zero; the validity of this assumption diminishes away from the center wavelength.

$$
n_{e f f}(\lambda)=\frac{1}{\lambda_{0}} \int\left(\left.n_{g}\right|_{\lambda_{0}}+\left(\lambda-\lambda_{0}\right) \frac{\delta n_{g}}{\delta \lambda}\right) \delta \hat{\lambda}
$$

The group index and the effective index are used in design inputs when the required functional parameters of FSR and phase of power coupling have to be converted in physical design parameters (width, length, gap, ...) . Equation (2) is used for designing the physical delay length starting from the group index and the required free spectral range. Equation (3) expresses the delay length to add in case $\pi$ shift is required.

$$
\begin{gathered}
\Delta L=\frac{\lambda_{0}^{2}}{n_{g} F S R} \\
\Delta l_{\pi}=\frac{\lambda_{0}}{2 n_{e f f}}
\end{gathered}
$$

The specifications obtained for the waveguide are then used to calculate the S-matrix of the circuit model, as well as the physical layout definition of the filter circuit. 


\subsection{Directional Coupler}

The directional coupler is the other basic block used in the lattice filters. It is basically composed of pair of waveguides that are optically coupled. The coupling $\mathcal{K}$ between the two waveguides is a result of the beating of the even and odd supermodes of the coupled parallel waveguide system. The stronger the waveguides are coupled, the larger the coupling factor $\kappa$ which will transfer power from one waveguide and back. The contribution of this mechanism varies sinusoidal along the length of the directional coupler. An additional contribution $\kappa_{0}$ to the overall coupling comes from the transition region were the waveguides come closer together and change regime from optically uncoupled to optically coupled.

$$
\left.\mathcal{K}=\sin \left(\left(\kappa(\lambda)+\left(\lambda-\lambda_{0}\right) \frac{\delta \kappa}{\delta \lambda}\right)\right) L+\left(\kappa_{0}(\lambda)+\left(\lambda-\lambda_{0}\right) \frac{\delta \kappa_{0}}{\delta \lambda}\right)\right)^{2}
$$

The contribution of the transition section is independent of the length, and will be identical (or very similar) for all couplers in our filter. It depends on the bending radius and on the gap separating the waveguides. For this work both gap and bending radius are designed to be the same for all couplers and fixed to 5um and $180 \mathrm{~nm}$, respectively. The first value guarantees low reflection of the bends and contained footprint, the latter gives sufficiently strong coupling for short couplers, and within the limits of the SOI fabrication platform. Equation (4) describes the model used to describe the directional coupler, it contains the sinusoidal relation that relates the power splitting and the length of the coupler. The coupling $\kappa$ is expressed in $1 / u m$ and is multiplied by the length, while the $\kappa_{0}$ is independent of the length and for this reason is dimensionless. Of course, just like $n_{\text {eff }}$, these quantities are wavelength dependent. Therefore, we expand them as a first order Taylor series in wavelength. This approach allow the model to include the linear wavelength dependency of the coupling parameters. Each of these coefficients becomes an independent model parameter. As we will show later, this proves to be sufficiently accurate, but if required, we could have added higher-order Taylor coefficients in our analysis.

\section{PROCESSING AND MEASUREMENTS}

\subsection{Processing}

The devices are processed through the ePIXfab MPW (multi-project wafer) service in IMEC, Belgium. The processing is carried out in CMOS pilot line on $200 \mathrm{~mm}$ SOI wafers with thin silicon $(220 \mathrm{~nm})$ on top of buried $2 u m$ silicon oxide. For the waveguides used in this work, a deep etch is used, with the full $220 \mathrm{~nm}$ of silicon etched around the waveguide core. On top of the waveguides a planarized silicon oxide is deposited.

\subsection{Measurements}

The full wafer is measured with an automated setup that measure a set of devices across the full wafer without interruptions. This keeps the measuring conditions stable for all the dies on the wafer. For each die, a reference structure is measured prior to the lattice filters of interest. The reference structure consists of a straight waveguide of a well known length connected to two grating couplers. ${ }^{11}$ The same vertical coupling device is used for the coupling of the lattice filter. The grating coupler has a wavelength-dependent response, but we normalize all our measurements to the response of the reference to eliminate most of the grating coupler effects. Alternatively, we could have considered the grating couplers to be part of our circuit to be analyzed.

The reference structure is replicated on each die close to the filter under test to assume that the processing variability affecting the grating couplers from reference structure and filter under test is negligible. This is not true on wafer scale, where the variability of wafer thickness and fabricated line widths changes the performance not only of the filter (this is what we are interested in) but the grating coupler performance as well, shifting the peak of the gaussian like response and its insertion loss. Therefore, we always normalized to a local reference structure on the same die. 


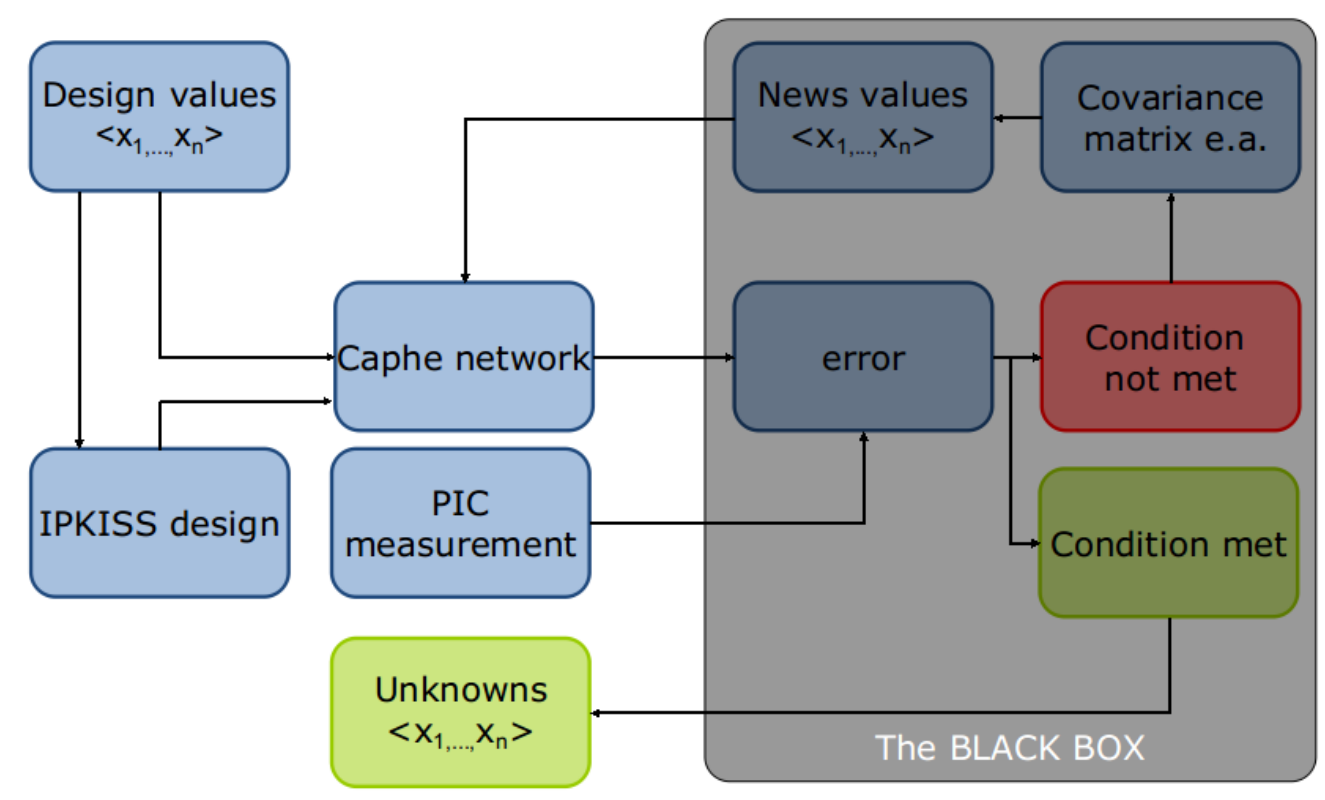

Figure 3: Algorithm block diagram

\subsection{Relevant Parameters}

The result of the measurement is the spectral response of the filter between different combinations of inputs and outputs. We did not collect traces of the backreflections; we assume these to be negligible. The optical response of the filter can now be used to extract the optical model parameters of the different sub-blocks, such as the waveguide group index, the coupling coefficients and their derivatives (wavelength dispersion). As expressed before, the group index is directly related to the FSR of the filter, while the coupling coefficients are related to the shape of the filter response within one FSR. The dispersion of the group index and the coupling coefficients shows up in differences of the stop-band shape in the different FSRs of the response. Because of variability across the wafer of silicon thickness and waveguide line width, the behavior of the filter changes from die to die, and this leads to a map of different functional performance. From such a wafer map we can deduce the variability of the optical properties of the devices, and correlate these properties with the process data collected during fabrication. The extracted model parameters, as well as the variability on their values, can then be used in the next iteration of the design.

\section{PROCEDURE SUGGESTED: THE BLACK BOX APPROACH}

Instead of using test structures for each individual sub-block, we extract the parameters directly from a device under test, in our case the multistage lattice filter. The easier approach to achieve this is to isolate the specs of the measured spectrum relative to the quantity we are interested in. For example, we know that the FSR is a direct measure for the group index of the delay lines. But such direct relations are not always known, if present at all. For instance, it is much more difficult is identify a signature in the spectrum that corresponds to the coupling coefficient: all values simultaneously affect the output of the filter. In our approach we look for a full convergence of the measured curve to the simulation of the photonic circuit instead of using two or few points evaluation. This has the advantage that we have much more data at our disposal, which makes fitting more reliable. Our objective function is the overall error between the measurement curve and the simulated curve: defined as the quadratic square root of the measured spectrum and of the simulated point by point. Instead of using analytical fitting techniques, ${ }^{12}$ the simulation module used for the parameter extraction is the same as the one used in phase of designing, using the same models for the circuit building blocks. This guarantee homogeneity between what has been designed and what is analyzed, and enables a direct reuse of the models for future designs. 


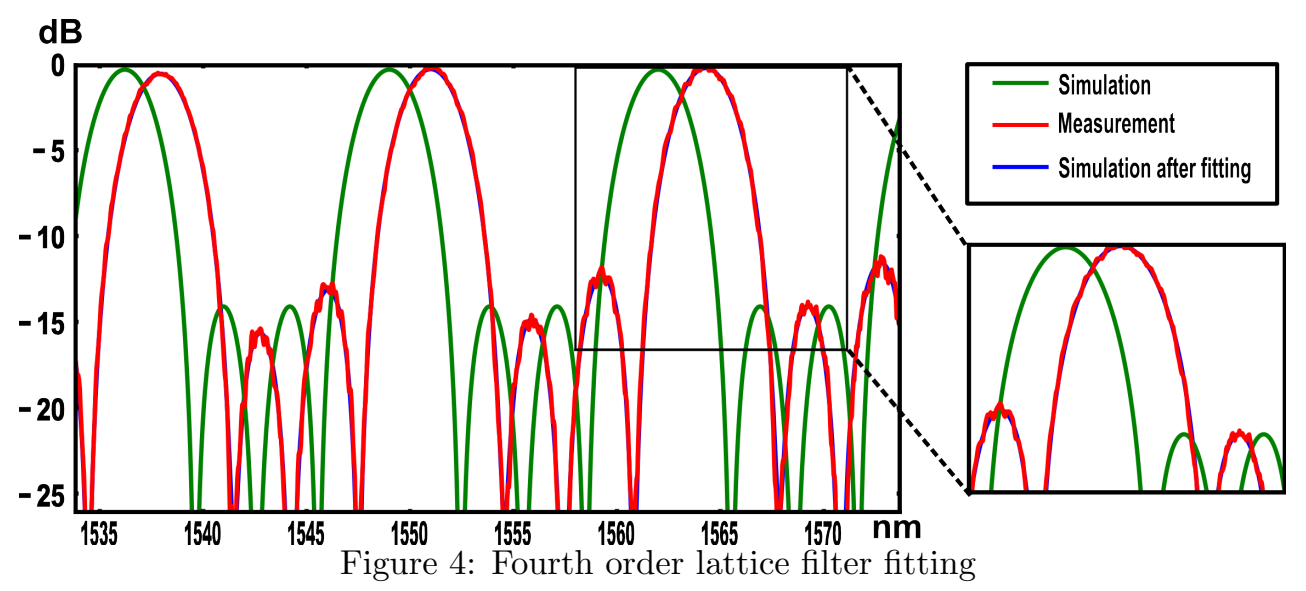

\subsection{Reuse of the Simulation Model Used for the Design}

What we aim to extract with our procedures are the unknown parameters of our behavioral models. During the design, we had kept these optical properties fixed while we optimized the physical parameters to achieve the required performances. During the parameter extraction phase, we keep the physical parameters are fixed at the values used in the final design, and the variables are the optical properties such as group index and coupling coefficients. The total circuit model is a hierarchical photonic integrated circuit with multiple unknowns in the models of the sub-blocks. Because we aim to minimize entire wavelength responses, a direct search for the convergency between the measured curve and the simulated one with brute force simulation is too computationally expensive, since the number of simulation required grows with the power of variables. We propose the use of an ES (Evolutionary Strategy) to dynamically rearrange the domain of exploring the variables. We selected the CMA (Covariance Matrix Adaptation) algorithm. ${ }^{13}$ The CMA method allows to cope with non-linear, not separable, non convex, noisy and with high condition number functions. This make it very suitable for targeting our measured spectrum. On the other hand, this method requires a fairly large number of simulations compared to other evolutionary strategies, but it avoids characteristic problems such as bad performances with small populations and premature convergence to local minima. The CMA optimization starts from a user defined population.

\subsection{Considering the circuit model as a black box}

The generalization of the optimization algorithm allows us to treat the model of the circuit as a black box. Is not strictly required to know what is in the sub-blocks of the circuit. We only require sufficiently descriptive models for the waveguides and directional couplers, and the description of the circuit connectivity. The measured spectrum is then fed to the circuit simulator specifying only the variables required to be fit. At the end of each simulation the evolutionary strategy uses the evaluation of error to evaluate the new test individuals and new step size. The speed of CAPHE circuit simulator allows convergence in the order of minutes for the device under test, using a powerful, but regular PC. Fig.3 represent the process diagram of the suggested procedure. Because the optimization algorithm does not concern itself with the actual simulation, the simulation is considered as a black box. The outcome of the loop is the set of best fit parameters.

As input we require the CAPHE network, the measured spectrum and a set of guess values for the unknowns. As initial values, we pick the original simulation values used for the design itself. Fig. 4 represent a particular case of a single lattice filter analysis. The starting simulation (green) is not matching with the measured curve (red) in position and shape. After the fitting, a simulation with the resulting set of model parameters (blue curve) gives an almost perfect fit. Over the full wafer, we obtain a mean value of the quadratic error of 0.0007 , and this includes the measurement noise.

\section{RESULTS AND COMMENTS}

The outcome of this procedure are the optical properties of the waveguide and of the directorial coupler. However, we still need to critically analyze the results. First of all, not all model parameters can be reliably extracted. 

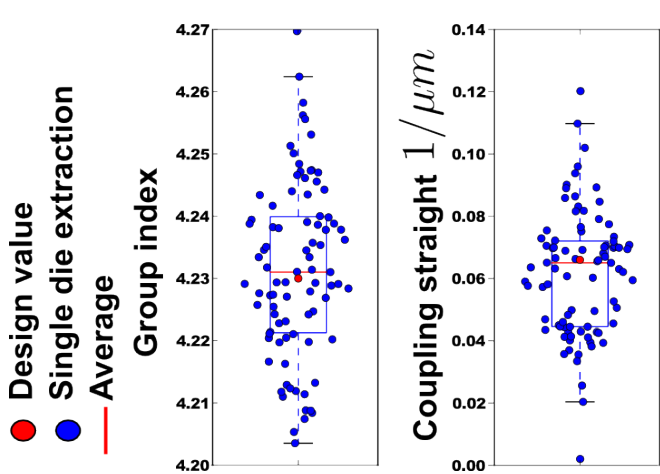

(a) Statistical results

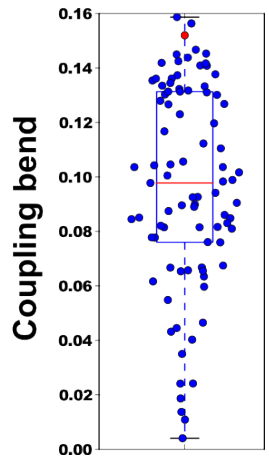

Figure 5: Extraction results

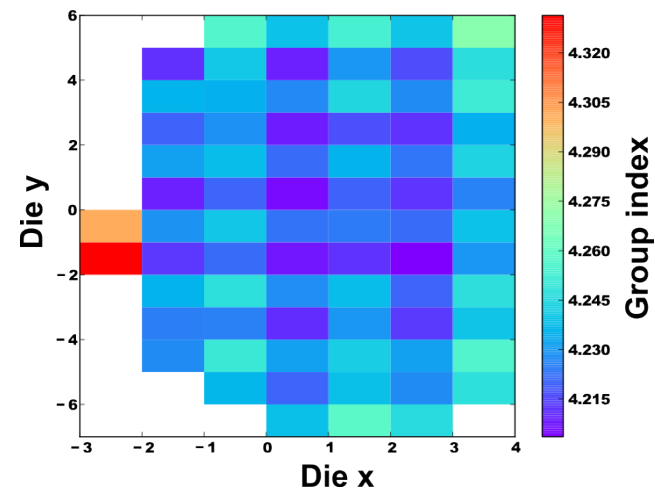

(b) Wafer map of group index

For instance, the effective index $n_{\text {eff }}$ cannot be easily extracted from a higher-order filter because it can only be determined up to an unknown constant. On the other hand, the extraction of the group index $n_{g}$ converges always to the same value for each die and converges always across the wafer. For the directional couplers, the coupling $\kappa_{0}$ due to the bend and transition section also converges properly. All of the quantities aforementioned converge to values in substantial agreement results of the simulation used for designing as well as to values obtained with individual testing structures of waveguides and directional couplers. The advantage in this case is that we can characterize directly the circuit and not a separate structure, saving space and avoiding local variability effects. Even more important is the wafer scale characterization. It can give important feedback on the performance of the processing and it can be trimmed to reach higher stability.

The results of such characterization can directly be fed as input into the models for future designs, improving the predictability of the technology platform. The variability instead can be used for prediction of the performance range after processing. Also, the same black-box approach can be used as a design tool for complex filter implementation: a practical example are ARMA (Auto Regressive Moving Average) filters. ${ }^{14,15}$ This family of devices allows non-unique analytical solutions for a design procedure. Using the ideal target curve instead of the measurement, a semi-empirical solution can be found for the physical properties such as delay length and coupler lengths. Fig.5 show the main outcome of the full wafer analysis and statistics. In detail Fig.5a shows the statistical results of the group index extracted from the filter. Except for the value to be attributed to border effects, the values match the results of the simulations. Also for the coupling coefficients of the straight section $\kappa$ and the coupling due to bend section section $\kappa_{0}$, as in Equation (4), the extracted values match the results of the simulation. Fig.5b represents the group index over the whole wafer. The obtained results can be correlated with the physical characteristics of the processed waveguides. The correlation between linewidth and thickness is known, but since no full information on both these quantities are known across the whole wafer, the reconstruction of the full correlation is not straightforward. The information on the wavelength derivatives extracted with the procedure, respect the expected trends both regarding the group index and the coupling, indeed their derivative are positive as expected. Their quantitative validity is compromised by the small range of analysis and by the overposition effect of the multiple grating coupler.

\section{CONCLUSIONS}

We presented a robust technique for fast photonic integrated circuits characterization. We tested the procedure on multistage lattice filters. The circuit has been simulated with CAPHE circuit simulator, designed with IPKISS, processed through ePIXfab and measured on wafer scale with our automated measurement setup. The measured spectrum has been used as input for the characterization tool leveraging together the speed of our circuit simulation and the fast convergence of CMA-ES. The results obtained have been compared with the values used for designing, this both to check the robustness of our method and to collect statistical data on the quality of our processing fab. 


\section{REFERENCES}

[1] Ruocco, A., Van Thourhout, D. V., and Bogaerts, W., "Soi lattice filters design framework: from functional parameters to layout," Proceedings of the 18th Annual Symposium of the IEEE Photonics Society Benelux Chapter, 223-226 (2013).

[2] Selvaraja, S., Jaenen, P., Bogaerts, W., Dumon, P., Van Thourhout, D. V., and R., B., "Fabrication of photonic wire and crystal circuits in silicon-on-insulator using 193nm optical lithography," Journal of Lightwave Technology 27(18), 4076-4083 (2009).

[3] Bogaerts, W., Li, Y., Pathak, S., Ruocco, A., Fiers, M., Ribeiro, A., Lambert, E., and Dumon, P., "Integrated design for integrated photonics: from the physical to the circuit level and back," Proc. of SPIE Vol 8781, 878102 (May 2013).

[4] Selvaraja, S., Bogaerts, W., Dumon, P., Thourhout, D. V., and Baets, R., "Sub-nanometer linewidth uniformity in silicon nano-photonic waveguide devices using cmos fabrication technology," IEEE Journal on Selected Topics in Quantum Electronics 16(1), 316-324 (January 2010).

[5] Dwivedi, S., D'heer, H., and Bogaerts, W., "Fabrication tolerant silicon mzi filter," International Conference on Group IV Photonics (GFP), 147-148 (2014).

[6] Horst, F., Green, W. M., Assefa, S., Shank, S. M., A, Y., Vlasov, and Offrein, B. J., "Cascaded machzehnder wavelength filters in silicon photonics for low loss and flat pass-band wdm (de-)multiplexing," Optics Express 21(10), 11652-11658 (2013).

[7] Bogaerts, W., De Heyn, P., Van Vaerenbergh, T., De Vos, K., Selvaraja, S., Claes, T., Dumon, P., Bienstman, P., Van Thourhout, D., and Baets, R., "Silicon microring resonators," Lasers \&f Photonics Reviews 6(1) (2012).

[8] Fiers, M., Lambert, E., Pathak, S., Dumon, P., Maes, B., Bienstman, P., and Bogaerts, W., "Improving the design cycle for nanophotonic components," Journal of Computational Science 4(5), 313-324 (2013).

[9] Bogaerts, W., Dumon, P., Lambert, E., Fiers, M., Pathak, S., and Ribeiro, A., "Ipkiss: A parametric design and simulation framework for silicon photonics," 9th International Conference on Group IV Photonics , p.30-32 (August 2012).

[10] Fiers, M., Vaerenbergh, T. V., Caluwaerts, K., Dambre, J., and Bienstman, P., "Caphe: Time-domain and frequency-domain modeling of nonlinear optical components," Advanced Photonics Congress IM2B.3 (June 2012).

[11] Taillaert, D., Van Laere, F., Ayre, M., Bogaerts, W., Van Thourhout, D., Bienstman, P., and R., B., "Grating couplers for coupling between optical fibers and nanophotonic waveguides," Japanese Journal of Applied Physics Vol 45, 6071-6077 (Aug 2006).

[12] Chen, X., Li, Z., Mohamed, M., Shang, L., and Alan, R., "Parameter extraction from fabricated silicon photonic devices," Applied Optics 53(7), 1396-1405 (2014).

[13] Hansen, N. and Ostermeier, A., "Adapting arbitrary normal mutation distributions in evolution strategies: the covariance matrix adaptation," Proceedings of IEEE International Conference on Evolutionary Computation, 312-317 (1996).

[14] Wang, Z., Chang, S., Ni, C., and Y.J., C., "A high-performance ultracompact optical interleaver based on double-ring assisted machzehnder interferometer," Photonics Technology Letters 19(14), 1072-1074 (2007).

[15] Song, J., Fang, Q., Tao, S., Yu, M., Lo, G., and Kwong, D., "Passive ring-assisted mach-zehnder interleaver on silicon-on-insulator," Optics Express 16(12), 8359-8365 (2008). 